\title{
Tumor 5-FU-related mRNA Expression and Efficacy of Oral Fluoropyrimidines in Adjuvant Chemotherapy of Colorectal Cancer
}

\author{
KEIJI KODA ${ }^{1}$, HIDEAKI MIYAUCHI ${ }^{2}$, CHIHIRO KOSUGI ${ }^{1}$, TAKASHI KAIHO ${ }^{3}$, NOBUHIRO TAKIGUCHI ${ }^{4}$, \\ SUSUMU KOBAYASHI ${ }^{5}$, TAKASHI MARUYAMA ${ }^{6}$ and HISAHIRO MATSUBARA ${ }^{2}$ \\ (Boso Clinical Oncology Group) \\ ${ }^{1}$ Department of Surgery, Teikyo University Chiba Medical Center, Chiba, Japan; \\ ${ }^{2}$ Department of Academic Surgery, Graduate School of Medicine, Chiba University, Chiba, Japan; \\ ${ }^{3}$ Department of Surgery, Kimitsu Chuo Hospital, Chiba, Japan; \\ ${ }^{4}$ Department of Surgery, Chiba Prefectural Cancer Center, Chiba, Japan; \\ ${ }^{5}$ Department of Surgery, Chiba Prefectural Sawara Hospital, Chiba, Japan; \\ ${ }^{6}$ Department of Surgery, Funabashi Municipal Medical Center, Chiba, Japan
}

\begin{abstract}
Background: It has not been elucidated whether the clinical efficacy of oral fluoropyrimidines for adjuvant chemotherapy of colorectal cancer varies with tumor biological characteristics. Patients and Methods: A multicenter randomized trial was performed comparing oral tegafur/gimeracil/oteracil (S-1) and uracil-tegafur/ leucovorin (UFT/LV) as adjuvant therapy for stage III colorectal cancer. Postoperative survival was compared based on the 5-FUrelated $m R N A$ levels in cancer tissues. Results: Among patients with tumor expressing dihydropyrimidine dehydrogenase (DPD) $m R N A$ within the 66.7th percentile (lower 2/3) of all cases, overall survival (OS) was significantly better in the $S-1$ than in the UFT/LV group. In the $S$-1 group, patients with low $D P D$-expressing tumors had significantly better OS than those with highly expressing tumors. Patients with low thymidine synthase (TS)-expressing tumors had significantly better OS than those with highly expressing tumors. Conclusion: The efficacy of oral fluoropyrimidines as adjuvant chemotherapy for colorectal cancer may be influenced by the level of 5-FUrelated $m R N A$ in cancer tissues.
\end{abstract}

This article is freely accessible online.

Correspondence to: Keiji Koda, MD, Ph.D., Professor and Chairman, Department of Surgery, Teikyo University Chiba Medical Center, 3426-3 Anesaki, Ichihara City, Chiba 299-0111, Japan. Tel: +81 436621211, Fax: +81 436613961, e-mail: kkoda@med.teikyo-u.ac.jp

Key Words: Colon cancer, rectal cancer, adjuvant therapy, S-1, uracil-tegafur, leucovorin, dihydropyrimidine dehydrogenase, thymidine synthase, 5-FU-metabolizing enzyme.
The postoperative survival of patients with primary colorectal cancer has been improving; a recent Japanese study reported 5-year disease-free survival (DFS) and overall survival (OS) rates for stage III colon/upper rectal cancer of $74.3 \%$ and $82.6 \%$, respectively, after adjuvant chemotherapy with 5fluorouracil (5-FU) and leucovorin (LV) over a 6-month period (1). Historically, oral fluoropyrimidines have been widely used postoperatively for 6-12 months as an adjuvant therapy for stage III colorectal cancer in Japan. A metaanalysis has shown the benefit of postoperative adjuvant therapy using oral fluoropyrimidines over surgery alone (2), as has a randomized controlled study (3). In many oral fluoropyrimidines, the mechanism of action is theoretically influenced by the content of modulators in the cancer tissue; such as thymidine phosphorylase (TP) in capecitabine (4), thymidine synthase (TS) or dihydropyrimidine dehydrogenase (DPD) in tegafur-uracil (UFT) or tegafur/gimeracil/oteracil (S-1). However, there have not been many clinical trials focusing on the efficacy of oral pyrimidines based on the content of modulators in cancer tissue. In the current study, we conducted a randomized clinical trial (UMIN000001560) comparing UFT/LV for 6 months with S-1 for 12 months for stage III colorectal cancers with a particular focus on mRNA expression of 5-FU metabolism-related enzymes in cancer tissue. Our aim was to determine whether the concentrations of these enzymes in cancer tissue can assist optimal selection of an oral anti-cancer fluoropyrimidine.

\section{Patients and Methods}

Patients and treatment protocol. Eligibility criteria were as follows: (i) histopathologically confirmed stage III adenocarcinoma, 
mucinous carcinoma or signet ring cell carcinoma of the colon and rectum; (ii) R0 resection; (iii) age 20-80 years; (iv) no previous treatment with chemotherapy or radiotherapy; (v) an ECOG performance status score of 0 or 1 ; (vi) adequate organ function (white cell count $\geq 3.5 \times 10^{9} / 1$ to $\leq 12.0 \times 10^{9} / 1$, neutrophil count $\geq 1.5 \times 10^{9} / 1$, platelet count $\geq 100 \times 10^{9} / 1, \mathrm{Hb} \geq 9.0 \mathrm{~g} / \mathrm{dl}$, aspartate aminotransferase and alanine aminotransferase $\leq 100 \mathrm{IU} / \mathrm{l}$, total bilirubin $\leq 2.0 \mathrm{mg} / \mathrm{dl}$, serum creatinine $\leq 1.2 \mathrm{mg} / \mathrm{dl}$ ); and (vii) patient's written informed consent. Patients who met the inclusion criteria were randomly assigned centrally to either the UFT/LV group or S-1 group. A minimization algorithm incorporating lymph node status, location of the tumor (colon $v s$. rectum) and performance status ( 0 vs. 1) was used to maintain a 1:1 balance within each Institute. In both groups, the doses of all drugs except for LV were determined according to the body surface area (BSA). For patients assigned to the UFT/LV group, UFT (300 mg with BSA $<1.17 \mathrm{~m}^{2} ; 400 \mathrm{mg}$ with BSA $1.17-1.49 \mathrm{~m}^{2}, 500 \mathrm{mg}$ with BSA 1.5$1.83 \mathrm{~m}^{2} ; 600 \mathrm{mg}$ with $\left.\mathrm{BSA}>1.83 \mathrm{~m} 2\right)$ and $\mathrm{LV}(75 \mathrm{mg}$ regardless of body weight) were administered orally three times a day (every 8 hours) at $>1$ hour before or after meals for 28 consecutive days, followed by a 7-day rest. This cycle was repeated five times for a total length of 25 weeks. For patients assigned to the S-1 group, S1 (40 mg with BSA $<1.25 \mathrm{~m}^{2} ; 50 \mathrm{mg}$ with BSA $1.25-1.5 \mathrm{~m}^{2} ; 60 \mathrm{mg}$ with BSA $>1.5 \mathrm{~m}^{2}$ ) was orally administered twice daily after meals for 28 consecutive days, followed by a 14-day rest. This cycle was repeated eight times for a total of 48 weeks. Assigned treatment was initiated within 8 weeks after surgery. The criteria for dose modification of UFT/LV and S-1 were in accordance with standard protocols used for colorectal cancer (1) and gastric cancer (5), respectively. The study protocol was approved by the Institutional Review Boards of all participating centers. Written informed consent was obtained from all participants before enrollment.

Follow-up. After registration in this trial, patients were followed up in accordance with a predefined protocol. Serum tumor markers were measured postoperatively every 3 months during the first 3 years, then every 6 months thereafter for $\geq 5$ years. Chest and abdominal computed tomography (CT) scans were performed every 6 months for the first 3 years and once a year thereafter. Additional CT scans were performed based on detection of increases in tumor markers. Colonoscopy was performed once a year for $\geq 5$ years. Recurrence was determined on the basis of diagnostic imaging.

Statistical analysis. The primary end-point of this study was to compare the 3-year DFS rate using the two treatment protocols. Secondary end-points were evaluation of OS, adverse events and the relationship between the expression of 5-FU-metabolizing enzymes in tumor tissue and prognosis. In accordance with the Japanese guidelines for treatment of colorectal cancer (6) and a meta-analysis of Japanese cases (2), the threshold and expected values for 3-year DFS were set at $60 \%$ and $75 \%$, respectively, with an $\alpha$ error of $5 \%$ in a one-sided test and a $\beta$ error of $10 \%$. The planned sample size was 140 ; there were 70 cases in each group, recruited during the 2 years from March 2008 to February 2010.

mRNA measurement of 5-FU metabolism-related enzymes. Expression of mRNA was measured using the quantitative reverse transcription polymerase chain reaction (PCR) in micro-dissected, formalin-fixed, paraffin-embedded tumor specimens and normalized to $\beta$-actin expression as the reference (the Danenberg tumor profile assay). The
Table I. Baseline characteristics of eligible patients.

\begin{tabular}{lccc}
\hline Characteristics & UFT/LV(n=71) & S-1(n=70) & $p$-Value \\
\hline Gender & & & \\
Male & 48 & 43 & \\
Female & 23 & 27 & n.s. \\
Age (year) & & & \\
Median & 68 & 69 & \\
Range & $38-79$ & $27-78$ & n.s. \\
Tumor location & & & \\
colon & 50 & 49 & \\
rectum & 21 & 21 & n.s. \\
Tumor stage & & & \\
T1 or T2 & 9 & 12 & \\
T3 & 49 & 44 & \\
T4 & 13 & 14 & n.s. \\
Nodal status & & & \\
N1a & 31 & 33 & \\
N1b & 24 & 24 & \\
N2a & 13 & 8 & \\
N2b & 3 & 5 & n.s. \\
Stage group & & & \\
IIIa & 8 & 11 & \\
IIIb & 56 & 63 & \\
IIIc & 7 & & \\
\hline & & & \\
\hline & $56 . s$. & \\
\hline
\end{tabular}

n.s., Not significant.

PCR conditions were $50^{\circ} \mathrm{C}$ for $10 \mathrm{~s}$ and $95^{\circ} \mathrm{C}$ for $10 \mathrm{~min}$, followed by 42 cycles at $95^{\circ} \mathrm{C}$ for $15 \mathrm{~s}$ and $60^{\circ} \mathrm{C}$ for $1 \mathrm{~min}$. The 5 -FUmetabolizing enzymes measured in this study were TS, DPD, TP, orotate phosphoribosyl transferase (OPRT) and dihydrofolate reductase (DHFR); all of these enzymes are related to the mechanism of action of either UFT/LV or TS-1 (Figure 1). UFT contains tegafur (a prodrug of 5-FU) and uracil; the latter is expected to competitively maintain in vivo 5-FU concentration. S-1 contains tegafur, gimeracil and oteracil in a molar ratio of 1:0.4:1. Gimeracil is a 100-fold more potent DPD inhibitor than the uracil in UFT. Oteracil potassium, an inhibitor of OPRT, is expected to inhibit the conversion of 5-FU into fluorodeoxyuridine monophosphate in the gut, thereby reducing gutassociated adverse effects. The level of expression of each enzyme gene was categorized using cut-offs at the 33.3rd, 50th or 66.7th percentiles (lower $1 / 3,1 / 2$, or $2 / 3$ ) in all cases.

\section{Results}

Patients. Between March 2008 and August 2010, 145 patients from 22 centers were enrolled in this study. The accrual period was extended for 5 months to achieve the pre-set case number. Five years have passed since the last case was enrolled. After allocation to treatments, four cases were found to be ineligible because they had undergone non-curative resections according to either the operation record or pathology report; these cases were, therefore, eliminated from the study. Of the remaining 141 cases, 71 were allocated to the UFT/LV and 70 to the $S-1$ group. Baseline characteristics are summarized in Table I. There were no differences 
Table II. Adverse events according to treatment protocol.

\begin{tabular}{|c|c|c|c|c|}
\hline \multirow[b]{2}{*}{ no $(\%)$} & \multicolumn{2}{|c|}{ UFT/ LV (N=73) } & \multicolumn{2}{|c|}{$\mathrm{S}-1(\mathrm{~N}=72)$} \\
\hline & All grade & Grade $3 / 4$ & All grade & Grade $3 / 4$ \\
\hline White blood cell count & $5(7)$ & $0(0)$ & $11(15)$ & $0(0.0)$ \\
\hline Neutrophil count & $4(5)$ & $0(0.0)$ & $9(13)$ & $3(4)$ \\
\hline Hemoglobin level & $10(14)$ & $0(0.0)$ & $9(13)$ & $0(0.0)$ \\
\hline Platelet count & $5(7)$ & $0(0.0)$ & $10(14)$ & $0(0.0)$ \\
\hline AST & $7(10)$ & $\underline{3(4)}$ & $11(14)$ & $1(1)$ \\
\hline ALT & $10(14)$ & $\underline{4(5)}$ & $11(15)$ & $1(1)$ \\
\hline Serum total bilirubin & $14(19)$ & $0(0.0)$ & $12(17)$ & $2(3)$ \\
\hline Creatinine & $1(1)$ & $0(0.0)$ & $1(1)$ & $1(1)$ \\
\hline Diarrhea & $16(22)$ & $4(5)$ & $22(31)$ & $6(8)$ \\
\hline Mucositis (examination findings) & $6(8)$ & $1(1)$ & $11(15)$ & $0(0.0)$ \\
\hline Mucositis (function/symptom) & $9(12)$ & $0(0.0)$ & $12(17)$ & $0(0.0)$ \\
\hline Anorexia & $15(21)$ & $0(0.0)$ & $17(24)$ & $0(0.0)$ \\
\hline Nausea & $13(18)$ & $0(0.0)$ & $12(17)$ & $0(0.0)$ \\
\hline Vomiting & $2(3)$ & $1(1)$ & $5(7)$ & $0(0.0)$ \\
\hline Rash & $5(7)$ & $0(0.0)$ & $14(21)$ & $1(1)$ \\
\hline Pigmentation & $4(12)$ & $0(0.0)$ & $\underline{24(33)}$ & $0(0.0)$ \\
\hline Fatigue & $8(11)$ & $2(3)$ & $16(22)$ & $1(1)$ \\
\hline
\end{tabular}

Underline represents higher frequency when compared to another group. All cases, including ineligible cases, were analyzed. AST, Aspartate transaminase; ALT, alanine transaminase.

between the groups. Expression of mRNA was successfully measured in cancer specimens from 138 of these cases.

Safety. The rate of completion of the protocol treatment in terms of course number is summarized in Figure 2. Final completion rates for UFT/LV for 6 months (five courses) and S-1 for 12 months (eight courses) were $87.3 \%$ and $67.1 \%$, respectively. The postoperative completion rate at 6 months was $78.6 \%$ for S-1, which was slightly lower than for UFT/LV. The major reasons for discontinuation were recurrence of disease and adverse events. The adverse events in all 145 cases are summarized in Table II. Diarrhea was a common toxicity using both protocols; grade 3/4 diarrhea occurred in $5 \%$ and $8 \%$ of patients receiving UFT/LV and S1 , respectively. Grade $3 / 4$ increases in aspartate transaminase/alanine transaminase occurred more frequently in the UFT/LV group, whereas grade $1 / 2$ rash and pigmentation occurred more frequently in the $S-1$ group. No treatment-related deaths occurred in either group.

Prognosis. Five-year DFS and OS rates in all eligible cases were $71.3 \%$ and $83.9 \%$, respectively. Figure 3 shows DFS and OS rates according to treatment protocol. The 5-year DFS rates in the UFT/LV and S-1 groups were $66.9 \%$ and $75.7 \%$, respectively; however, this difference was not significant. The 5-year OS rates were $79.5 \%$ and $88.2 \%$, respectively. However, the tendency for the S-1 group to have a higher OS rate was not significant.
Next, correlations between expression levels of mRNA of 5-FU-metabolizing enzymes and prognosis were investigated for each treatment protocol. Among the 138 cases whose tumor $D P D$ mRNA could be successfully measured, the 5year OS was significantly higher in the S-1 group (93\%) than in the UFT/LV group (77.8\%) in those patients whose DPD expression was within the 66.7th percentile (lower 2/3) among all cases $(n=92 ; p=0.04)$; however, the DFS rate did not differ significantly (Figure 4). The OS rate was also higher in the S1 group than in the UFT/LV group among cases with $D P D$ expression within the lower half of all cases $(n=69 ; p=0.027$; data not shown). Among patients treated with $\mathrm{S}-1$, cases with tumors expressing low DPD mRNA (categorized as lower than the 50th percentile) had a significantly higher OS rate than those with high DPD expression (97.0\% vs. $71.2 \%$; $p<0.01$; Figure 5A). In contrast, in the UFT/LV group, no such tendency was identified (Figure 5B).

Among the 138 cases whose tumor TS mRNA could be successfully measured, those with tumors with low TS mRNA expression had a significantly higher OS rate than those with high TS expression (Figure 6A) (categorized as below and above the 50th percentile, respectively). This tendency was seen in both treatment groups but was more pronounced in the UFT/LV than the S-1 group. In the UFT/LV group, cases with low TS expression (when categorized as below the 50th percentile) tended to have a higher OS rate than those with high TS expression ( $p=0.054$, Figure 6B). No other correlations were found between the 
UFT (tegafur, uracil)

S-1 (tegafur, gimeracil, oteracil)
Inhibitor

Target enzymes measured

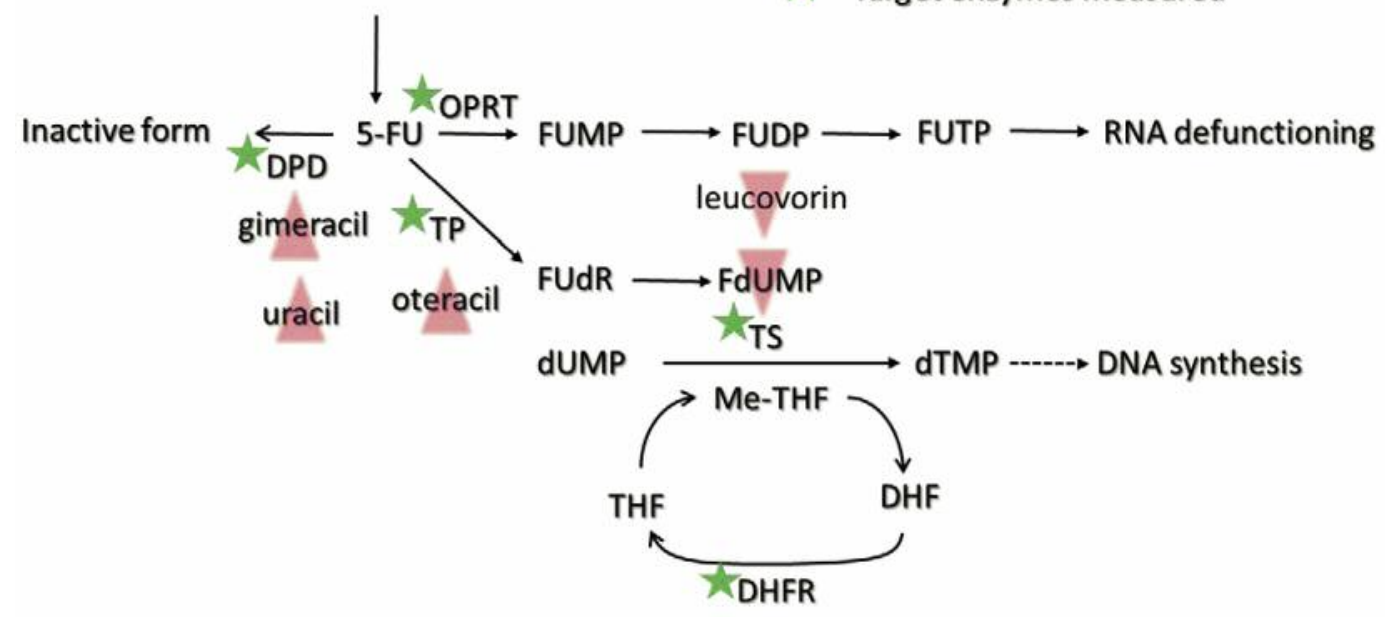

Figure 1. Mechanisms by which fluoropyrimidine compounds interfere with DNA/RNA synthesis. 5-FU, 5-Fluorouracil; OPRT, ortate phosphoribosyl transferase; FUMP, 5-fluorouridine monophosphate; FUTP, 5-fluorouridine triphosphate; TP, thymidine phosphorylase; FUdR, 5-fluorodeoxyuridine; FdUMP, 5-fluorodeoxyuridine monophosphate; FdUTP, 5-fluorodeoxyuridine triphosphate; TS, thymidine synthase; dUMP, deoxyuridine monophosphate; dTMP, deoxythymidine monophosphate; dTTP, deoxythymidien triphosphate; DPD, dehydropyrimidine dehydrogenase; DHFR, dehydropholate reductase; THF, tetrahydropholate; Me-THF, methyltetrapholate.

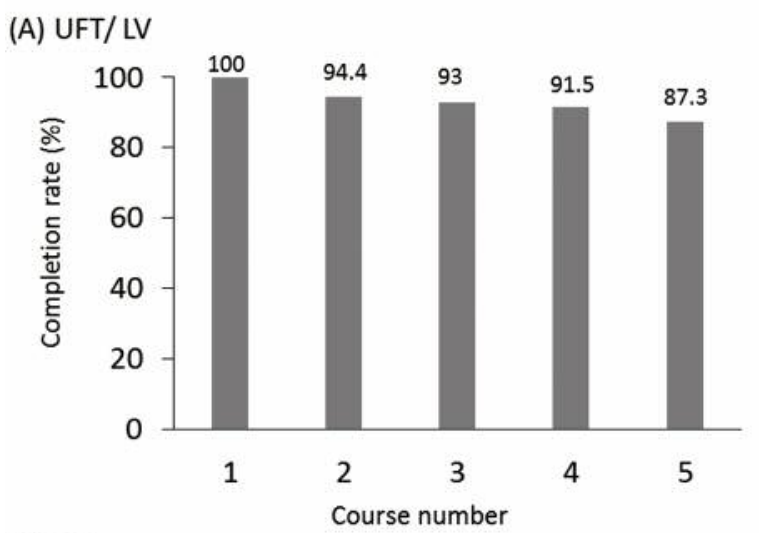

(B) S-1

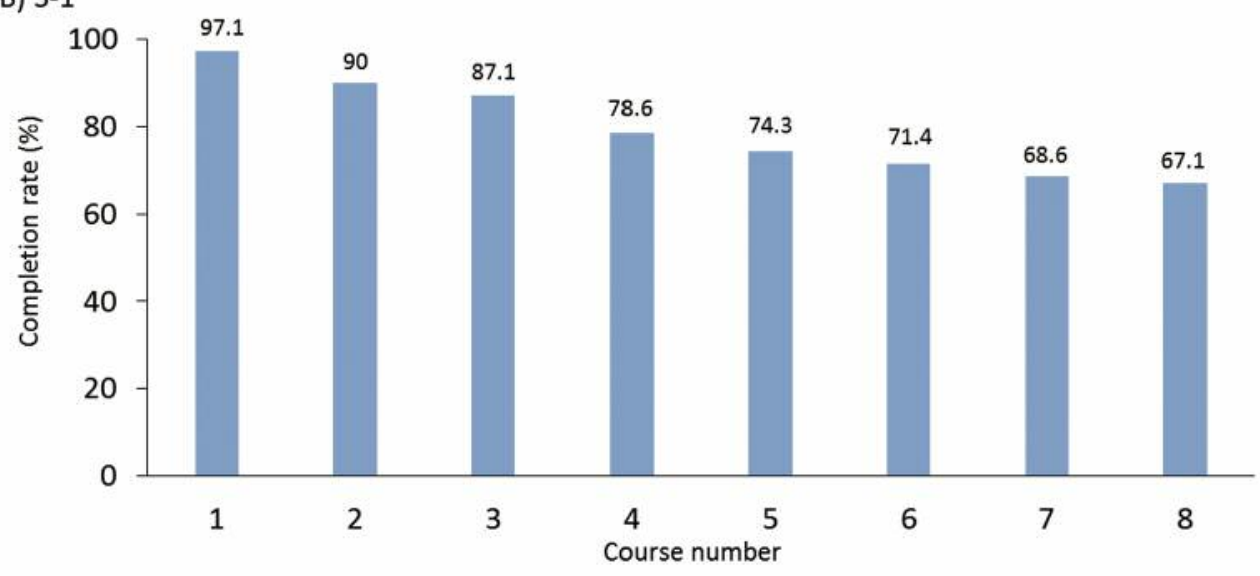

Figure 2. Rate of completion of protocol treatments by number of courses received. 
(A) DFS - all cases

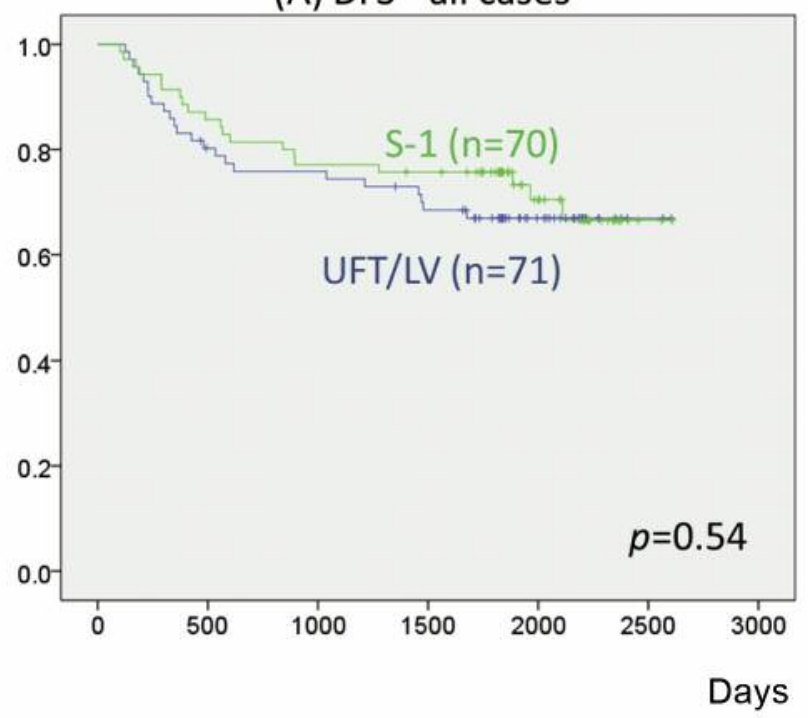

(B) OS - all cases

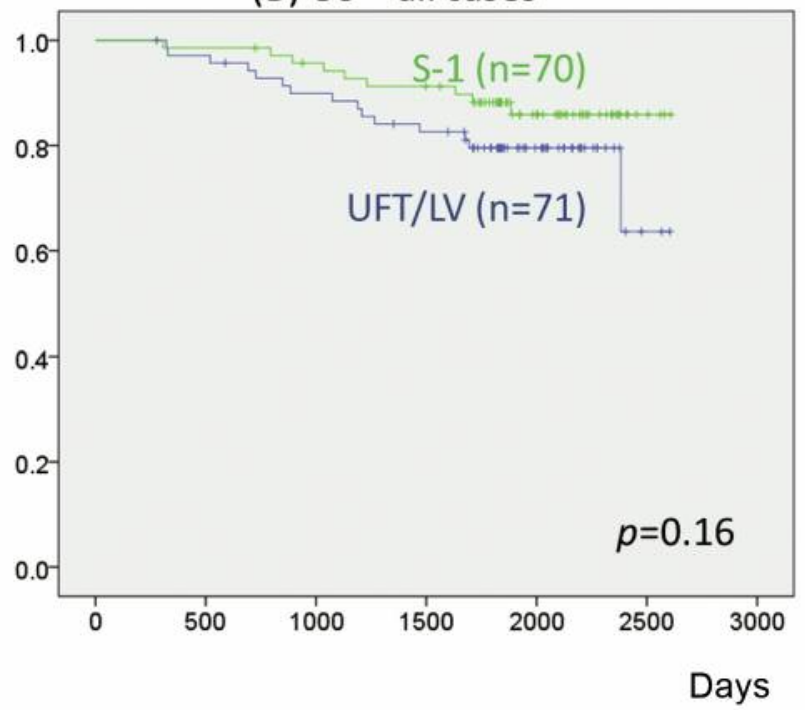

Figure 3. Postoperative survival rates according to the treatment received.

(A) DFS - DPD $\leq 67 \%$

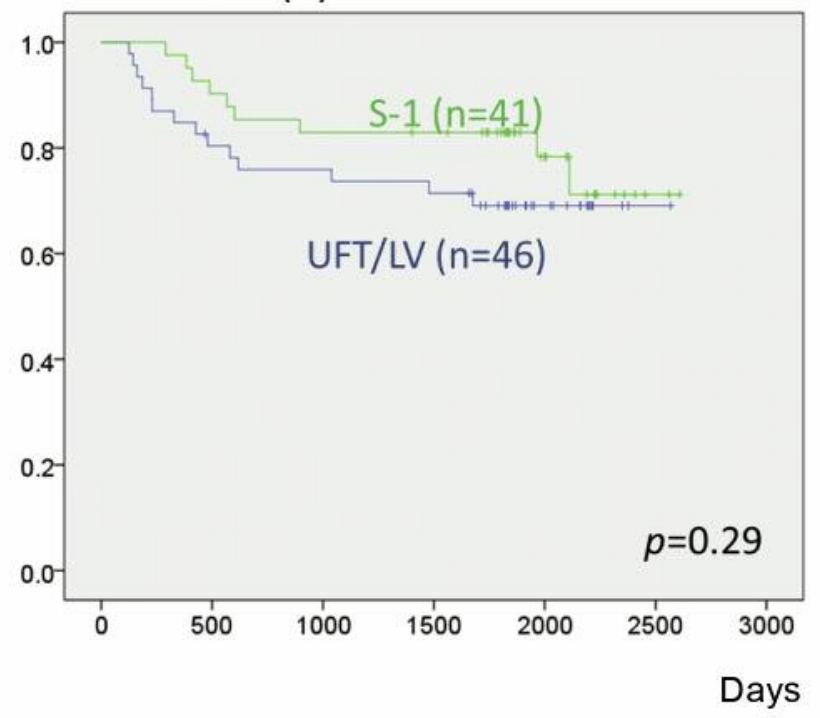

(B) $O S-D P D \leq 67 \%$

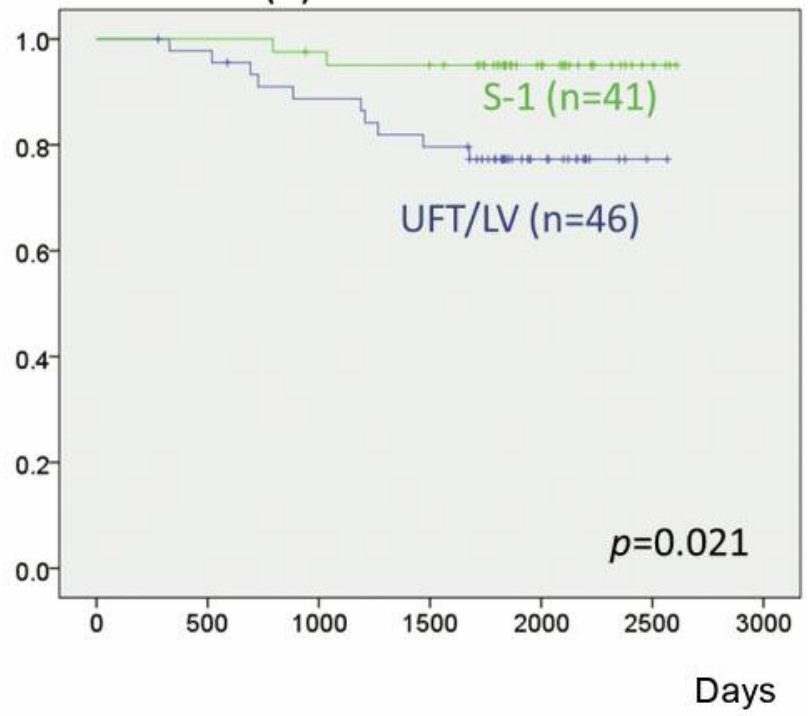

Figure 4. Survival rates according to the treatment received in patients with tumors expressing $<66.7$ th percentile (the lower $2 / 3$ ) of the DPD $m R N A$ level among all cases.

levels of 5-FU metabolism-related enzymes and prognosis according to treatment group.

\section{Discussion}

Historically, oral fluoropyrimidines administered for 6-12 months has been widely used for adjuvant therapy of stage
III colorectal cancer in Japan. At the start of this study, treatments with UFT/LV and 5-FU/LV for 6 months had been reported to be equally effective for adjuvant chemotherapy for both stage II and III colon cancer (7). However, there is no definite evidence for the efficacy of S-1 for adjuvant chemotherapy of colorectal cancer. Yet, S-1 has been reported to improve postoperative survival in stage II and III gastric 
(A) OS - S-1 group

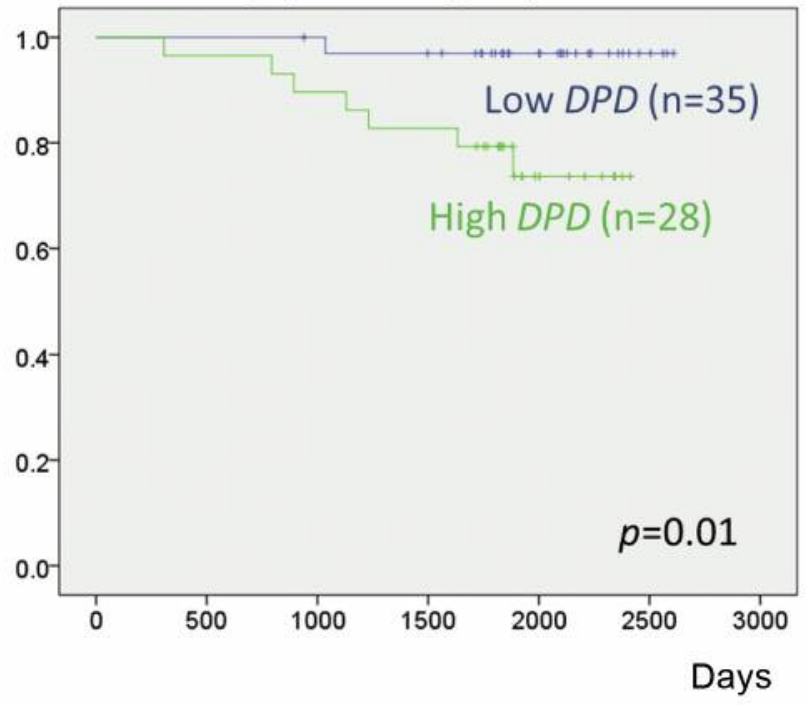

(B) OS - UFT/LV group

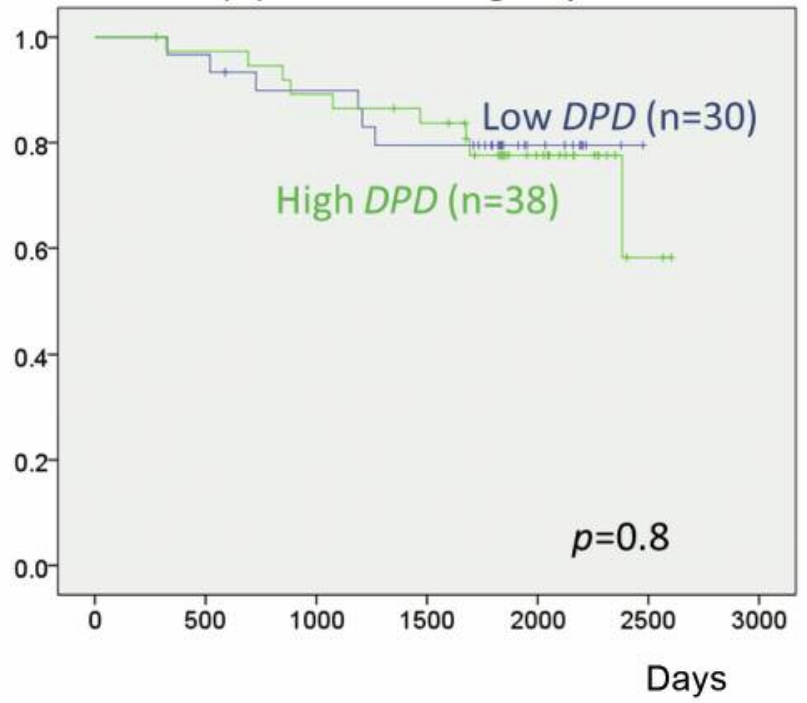

Figure 5. OS according to DPD mRNA levels. A: OS in S-1-treated patients according to DPD mRNA expression levels. B: OS in UFT/LV-treated patients according to DPD mRNA expression levels. DPD high, $\geq 50$ th percentile; DPD low, $<50$ th percentile in all cases.

(A) OS - all cases

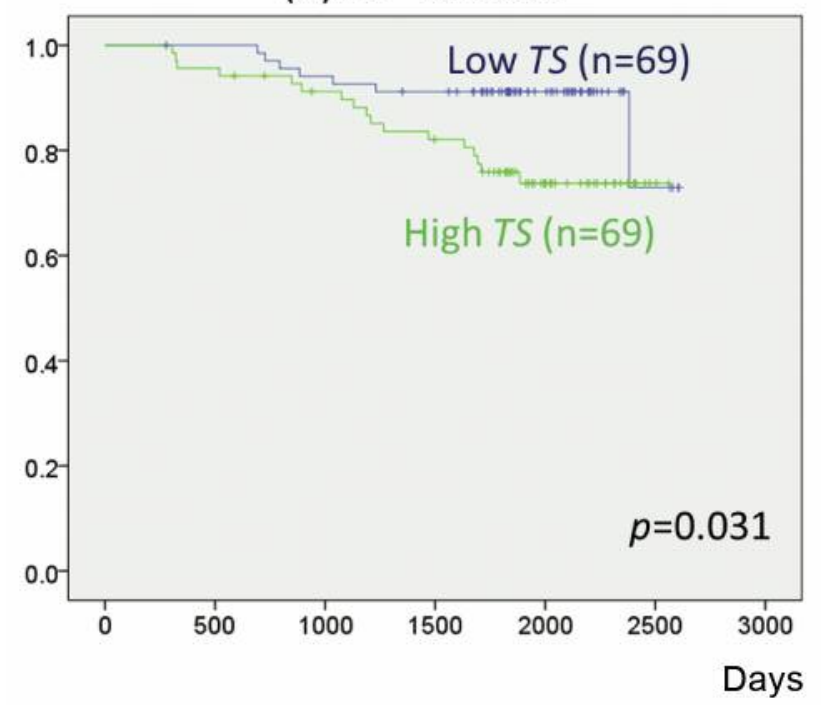

(B) OS - UFT/LV group

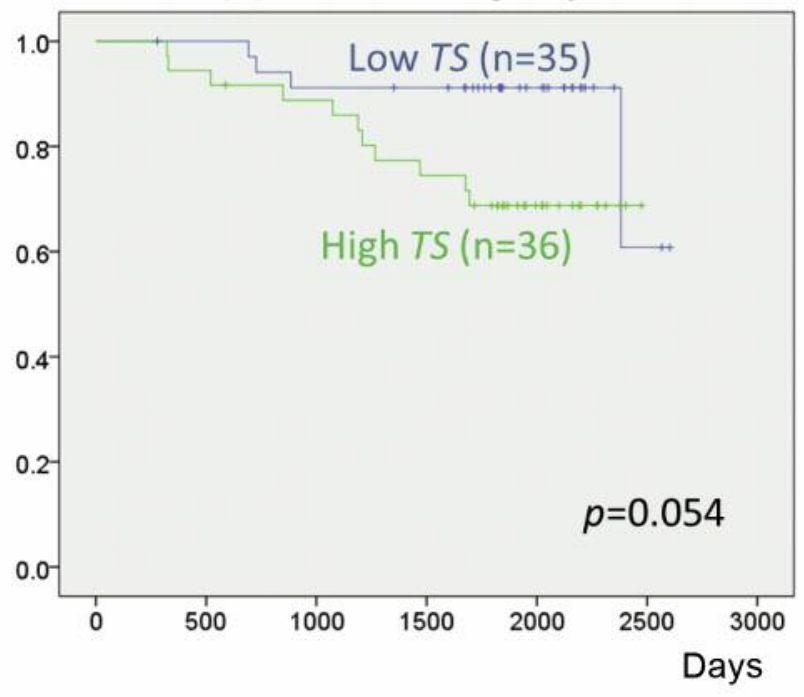

Figure 6. OS according to TS mRNA expression levels. A: OS in all cases according to TS mRNA expression levels. B: OS in UFT/LV-treated patients according to TS $m R N A$ expression levels. TS high, $\geq 50$ th percentile; TS low, $<50$ th percentile in all cases.

cancer when administered postoperatively for 1 year (8). Oxaliplatin-based regimens were unavailable for adjuvant therapy when the current study was initiated in 2008 .

In the current study, we investigated correlations between the level of mRNA expression of 5-FU-metabolizing enzymes in the tumor and the efficacy of oral UFT/LV and $\mathrm{S}-1$. The aim was to determine whether enzyme profiles can suggest the optimal oral chemotherapy agent. Because these agents contain modulators of 5-FU-metabolizing enzymes, postoperative prognosis may be influenced by the levels of 
these enzymes in the tumor. Our data showed that, among patients whose tumor expressed a low to moderate amount of $D P D$ mRNA (within the 66.7th percentage in all cases), the OS rate was significantly higher in the S-1 group than in the UFT/LV group. Additionally, among patients treated with $\mathrm{S}-1$, the OS rate was significantly higher in cases with low $D P D$ expression than in those with high expression. However, no such tendency was noted in patients treated with UFT/LV. These findings suggest that gimeracil within S-1 effectively inhibits the activity of DPD in degrading 5FU when the tumor expresses small to moderate levels of DPD. Other studies have reported that weak $D P D$ expression is beneficial in patients receiving adjuvant therapy with UFT alone for stage III colorectal cancer (9). The findings of the current study suggest that $\mathrm{S}-1$ is a good candidate for adjuvant therapy of stage III colorectal cancer when the tumor does not express high levels of $D P D$.

TS is one of the key target enzymes for the inhibition of DNA synthesis by 5-FU (Figure 1). Among the study subjects who received oral fluoropyrimidine, OS was significantly better in those with tumors with low $T S$ expression than in those with high $T S$ expression (Figure 6A). For treatment with UFT/LV, the enhancement of the inhibition of TS activity is thought to occur through the formation of a ternary complex consisting of a UFT degradation product (FdUMP), TS, and activated folic acid that originates from the LV regimen (Figure 1). There was a tendency for patients treated with UFT/LV, whose tumors had $T S$ expression less than the 50th percentile, to have better OS than those with higher TS expression (Figure 6B). This tendency was present but weaker in cases treated with S-1 (data not shown). These findings suggest that patients with tumors expressing small to moderate levels of $T S$ mRNA showed better OS than those expressing high levels of TS, when adjuvant chemotherapy was conducted using oral fluoropyrimidine, especially UFT/LV.

Although the data presented in the current study are limited because of the small number of cases, they suggest that S-1 is no less effective as adjuvant chemotherapy for stage III colorectal cancer than UFT/LV. In particular, when the tumor contains small to moderate levels of DPD mRNA, the gimeracil in S-1 may more effectively inhibit DPD and, thus, help to maintain 5-FU concentrations in vivo. Additionally, UFT and LV may work more effectively and synergistically to inhibit TS when tumors contain small to moderate amounts of TS. Modulation of 5-FU-metabolizing enzymes may help fluoropyrimidine work more effectively.

\section{Conflicts of Interest}

The Authors declare no conflicts of interest.

\section{References}

1 Shimada Y, Hamaguchi T, Mizusawa J, Saito N, Kanemitsu Y, Takiguchi N, Ohue M, Kato T, Takii Y, Sato T, Tomita N, Yamaguchi S, Akaike M, Mishima H, Kubo Y, Nakamura K, Fukuda $\mathrm{H}$ and Moriya Y: Randomised phase III trial of adjuvant chemotherapy with oral uracil and tegafur plus leucovorin versus intravenous fluorouracil and levofolinate in patients with stage III colorectal cancer who have undergone Japanese D2/D3 lymph node dissection: Final results of JCOG0205. Eur J Cancer 50: 2231-2240, 2014.

2 Sakamoto J, Hamada C, Yoshida S, Kodaira S, Yasutomi M, Kato T, Oba K, Nakazato H, Saji S and Ohashi Y: An individual patient data meta-analysis of adjuvant therapy with uracil-tegafur (UFT) in patients with curatively resected rectal cancer. Br J Cancer 96: 1170-1177, 2007.

3 Hamaguchi T, Shirao K, Moriya Y, Yoshida S, Kodaira S and Ohashi Y: Final results of randomized trial by the National Surgical Adjuvant Study of Colorectal Cancer (NSAS-CC). Cancer Chemother Pharmacol 67: 587-596, 2011.

4 Walco CM and Lindley C: Capecitabine: a review. Clin Ther 27: 23-24, 2005.

5 Yoshida M, Ishiguro M, Ikejiri K, Mochizuki I, Nakamoto Y, Kinugasa Y, Takagane A, Endo T, Shinozaki H, Takii Y, Mochizuki H, Kotake K, Kameoka S, Takahashi K, Watanabe T, Watanabe M, Boku N, Tomita N, Nakatani E and Sugihara K: S-1 as adjuvant chemotherapy for stage III colon cancer: A randomized phase III study (ACTS-CC trial). Ann Oncol 25: 1743-1749, 2014.

6 Japanese Society for Cancer of the Colon and Rectum (JSCCR) guideline 2005 for the treatment of colorectal cancer. Muto T (ed.), Kanehara Shuppan Co.,Tokyo, Japan.

7 Lembersky BC, Wieand HS, Petrelli NJ, O'Connell MJ, Colangelo LH, Smith RE, Seay TE, Giguere JK, Marshall ME, Jacobs AD, Colman LK, Soran A, Yothers G and Wolmark N: Oral uracil and tegafur plus leucovorin compared with intravenous fluorouracil and leucovorin in Stage II and III carcinoma of the colon: Results from National Surgical Adjuvant Breast and Bowel Project Protocol C-06. J Clin Oncol 24: 20592064, 2006.

8 Sasako M, Sakuramoto S, Katai H, Kinoshita T, Furukawa H, Yamaguchi T, Nishimoto A, Fujii M, Nakajima T, Ohashi Y: Five-year outcomes of a randomized phase III trial comparing adjuvant chemotherapy with S-1 versus surgery alone in stage II or III gastric cancer. J Clin Oncol 29: 4387-4393, 2011.

9 Mori T, Ohue M, Takii Y, Hashizume T, Kato T, Kotake K, Sato $\mathrm{T}$ and Tango $\mathrm{T}$ : Factors predicting the response to oral fluoropyrimidine drugs: A phase II trial on the individualization of postoperative adjuvant chemotherapy using oral fluorinated pyrimidines in stage III colorectal cancer treated by curative resection (ACT-01 Study). Oncol Reports 29: 437-444, 2013. 